\title{
Liver Transplantation
}

National Cancer Institute

\section{Source}

National Cancer Institute. Liver Transplantation. NCI Thesaurus. Code C15271.

The transfer of a healthy liver allograft from a donor to a patient. 